studies reviewed demonstrated potential benefits of introducing cuffed tubes into practice as they reduced exchange rate, air leaks and the costs associated with the anaesthetic gases used; without a significant increase in the rates of post-extubation stridor or failed extubation.

Conclusion In conclusion cuffed tubes are shown to decrease the need for multiple intubations, reduce costs and are not shown to increase adverse effects in children of all ages.

\section{P0-0914 POSTOPERATIVE HYPERALGESIA IN CHILDREN AFTER ONCOLOGY SURGERY CORRELATES WITH RAISED LEVEL OF CORTISOL, INTERLEUKIN 6 (IL6), IL8 (IL8) AND C-REACTIVE PROTEIN (CRP)}

D Dmytriiev. Intensive Care, Vinnitsa National Medical University, Vinnitsa, Ukraine

\subsection{6/archdischild-2014-307384.1535}

Background The mechanisms contributing to postoperative hyperalgesia $(\mathrm{PH})$ in children are multifactorial. Recent evidence suggests a potential pathogenetic role for inflammation.

Objective To examine the relationship between serum concentrations of inflammatory mediators, cortisol (hydrocortisone) and PA after oncology surgicy.

Methods Prospective observational study involving children with $\mathrm{PH}$ after oncology surgery and normal controls. All patients after operation received adequate analgesic therapy (continuous infusion opioid analgesics). Blood samples were taken at birth from peripheral venous blood, at $8 \mathrm{~h}, 24$ and $42 \mathrm{~h}$ for cytokines, cortisol and CRP after surgical procedure. The area of hyperalgesia for punctuate mechanical stimuli around the incision was measured $48 \mathrm{~h}$ after the operation with a hand-held von Frey filament. For statistical analysis 2 tests were used.

Results 26 children (12,2 +/- 2,4 years) with PA and 20 controls $(12,8+/-3,1$ years) were enrolled. 14/26 (53,8\%) children with $\mathrm{PH}$ required mechanical ventilation. Children with $\mathrm{PA}$ had more than threefold higher serum levels of interleukin 8 (IL8) than the controls $(\mathrm{p}<0.05)$. At $8 \mathrm{~h}, 24 \mathrm{~h}$ and $42 \mathrm{~h}$, serum IL6 and CRP were 2.43- fold higher in neonates than the controls group $(\mathrm{p}<0.003)$. All patients with $\mathrm{PH}$ had significantly $(\mathrm{p}<0.001)$ higher plasma cortisol levels over control group (mean $+/-\mathrm{SD}$, $464.42+/-56.40$ vs. $202.21+/-37.30 \mathrm{micromol} / \mathrm{l}$ on $8 \mathrm{~h}$; $752.02+/-96.4$ vs. $308.12+/-100.1 \mathrm{micromol} / \mathrm{l}$ on $24 \mathrm{~h}$; $600.04+/-62.3$ vs. $302.8+/-51.2 \mathrm{micromol} / \mathrm{l}$ in $42 \mathrm{~h}$ ).

Conclusion This study demonstrated that postoperative hyperalgesia syndrome is associated with raised blood levels of proinflammatory mediators and cortisol, suggesting that inflammation contributes to the PA.

\section{PO-0915 THERAPEUTIC OPTIONS FOR CONDUCTIVE HEARING LOSS IN CHILDREN WITH CLEFT PALATE}

${ }^{1} S$ Franchella, ${ }^{1} \mathrm{MR}$ Barillari, 'R Bovo, ${ }^{1} \mathrm{~A}$ Martini, ${ }^{2} \mathrm{~A}$ Franchella. 'University Hospital, Otosurgery Unit, Padua, Italy; ${ }^{2}$ Maternity and Childhood, Pediatric Surgery, Ferrara, Italy

10.1136/archdischild-2014-307384.1536

Background and aim Cleft lip and palate is a common congenital malformation correlated with otological disorders like
Eustachian tube dysfunction, otitis media with effusion and conductive hearing loss correlated with speech disorders.

Methods In our study we evaluate the therapeutic options for the conductive hearing loss in these children, such as: middle ear ventilation tube insertion, hearing aids and adenoidectomy.

We conducted a retrospective chart review on 19 patients who underwent last follow-up during 2013, aged between 1 and 16 , affected by cleft palate. We identified 3 cases of TreacherCollins syndrome, 2 cases of CHARGE syndrome, 2 case sof Pierre Robin, 1 case of Goldhenar and 1 case of Di George syndrome. In 9 cases the cleft palate was isolated. In 14 cases we conducted phoniatric and logopaedic evaluations and 10 of these showed a speech disorder.

Results We found a conductive hearing loss in 12 of the 19 children. 2 of these patients used hearing aids with an improvementof speech performance; 1 patient underwent adenoidectomy for the appearance of sleep apnea but he modified his quality of voice with hypernasality and nasal emission after surgery; finally 9 children had spontaneous resolution of otitis media with effusion.

Conclusions In conclusion we found that the more effective way to resolve the problem of conductive hearing loss and the resulting speech disorder is to use the hearing aids until the resolution of the hearing loss, which normally occurs around 7 years old.

\section{PO-0916 MANAGEMENT OF GIANT CONGENITAL MELANOCYTIC NEVI: A SINGLE CENTRE 20 YEARS EXPERIENCE}

E Cesca, A Cazzuffi, A Franchella. Maternity and Childhood, Pediatric Surgery, Ferrara, Italy

\subsection{6/archdischild-2014-307384.1537}

Background and aim Giant congenital melanocyticnevus $(\mathrm{GCMN})$ is a pigmented lesion present at birth with wide extent on the skin surface. This study aimed to assess the management of these lesions, which remains controversial and needs to take into consideration the perceived risk of melanoma, the patient's age, the cosmetic outcome, the surgical complexity, and the anesthesiological risk.

Methods This was a retrospective analysis and review of all records of children observed at the Paediatric Surgery Department oh the University-Hospital of Ferrara between 1991 and 2011 and treated for GCMN.

Results Twelve patients (median age 7 years, range 0.5-14) with GCMN were reported during the study period. Neurocutaneous melanosis was documented only once. Four patients underwent staged excision with grafting, two dermabrasion, and six implantation of skin dilator-expanded flap transfer. 2/12 lesions were macroscopically completely excised. Compliance to therapy was close to $100 \%$. metastatic melanoma from an unknown primary site and death occurred in 1/12 (mean follow-up, 14 years).

Conclusions Decision making process to produce a final treatment choice for GCMN can be really complex. Regardless the outcome of therapy, a close regular post-operative follow-upto prevent or exclude possible complications proved to be useful in children with GCMN. 